\title{
ОПРЕДЕЛЕНИЕ ПАРАМЕТРОВ ПЛАСТА НА ОСНОВАНИИ ИСЛЕДОВАНИЙ КРИВОЙ ИЗМЕНЕНИЯ ДАВЛЕНИЯ В УСЛОВИЯХ ГАЗОВЫХ СКВАЖИН ТУРКМЕНИСТАНА
}

\author{
Гелдимырадов Арслан Гелдимырадович \\ доктор технических наук, доцент Международного университета нефти \\ и газа имени Ягшигельды Какаева
}

Аннотация: с помощью эффективных исследований можно выяснить, что эффективные решения могут иметь положительное влияние на получение решений отрицательных проблем с более высоким разрешением. Повышая точность геолого-математической модели пласта, необходимо изыскать и реализовать лучший способ «фильтровать» рассматриваемые промысловые скважины, чтобы лучше «почувствовать» свойства коллектора и анизотропию проводимости пласта

Ключевые слова: исследования, фильтрация, коллекторские свойства пласта, пьезопроницаемость, давление, пласт, интегральный индекс.

В теории фильтрации особое внимание уделяется двум основным вопросам прямому и обратному. Прямые вопросы широко используются для прогнозирования динамики параметров эксплуатации скважин и работы месторождения с учетом известных параметров пласта и свойств жидкостей в пласте. Обратные моменты играют важную роль, связанные с определением и при решении задач исследований коллекторных свойств пласта на основе конкретных данных анализа или использования конкретной одной скважины, добывающей, наблюдательной и группы нагнетательных скважин. Первые и самые простые противоречия в истории часто связаны с интерпретацией результатов анализа проб. В результате выталкивания флюидов внутри образцов через поры определяются абсолютный коэффициент проводимости, анизотропия проницаемости и толщины пласта, зависимость соответствующей проницаемости от насыщенности отдельных фаз.

Понятно, что конкретные данные по эксплуатации нефтяных и газовых месторождений содержат информацию о коллекторских свойствах пласта и окружающего коллектора стоячей воды. Поэтому необходимо изучить 


\section{НАУКА, ОБЩЕСТВО, ТЕХНОЛОГИИ: ПРОБЛЕМЫ И ПЕРСПЕКТИВЫ ВЗАИМОДЕЙСТВИЯ В СОВРЕМЕННОМ МИРЕ}

интерпретацию конкретных данных по использованию скважин с целью «точного» выявления желаемых параметров пласта.

Нам уже известно, что все обратные вопросы являются неправильными вопросами. Например, небольшое изменение входных параметров (размеров) может привести к большому изменению решения (оцениваемых свойств пласта). Эффективные и надежные алгоритмы решения задач обнаружения должны соответствовать следующим условиям:

- процессы адаптации должны быть оптимизированы для получения оптимальных решений даже при использовании самых неблагоприятных начальных приближений;

- правило определения параметров пласта должно выполнять быстрое накопление при проведении итерации, а также при минимальных вычислительных затратах на поиск полного решения;

Необходимо урегулировать задачу определения минимального количественного правила и предотвратить образование решения, не имеющего физического смысла, и уменьшить его неточность.

Точность обнаружения задачи зависит от точности измеренных показателей работы, добывающей и нагнетательных скважин. Надежный алгоритм решения отрицательных проблем по указанным выше причинам требует, чтобы были получены только определенные (реальные) эквиваленты заданного набора параметров пласта.

Понятно, что точные ответы на волнующие нас вопросы можно получить только на тестовых примерах. В этом случае «точные» показатели производительности сначала получаются от компьютера на как можно более высоком уровне. Во-вторых, мы точно знаем, каковы требуемые значения, такие как свойства коллектора каждого уровня элемента. В-третьих, не вызывает сомнений точность значений кровли и подошвы пласта, а также конфигурации и размеров внешней границы пласта.

C помощью эффективных исследований можно выяснить, что эффективные решения могут иметь положительное влияние на получение решений отрицательных проблем с более высоким разрешением.

Это приводит к очень важному практическому выводу. Если мы хотим повысить точность геолого-математической модели пласта, нам необходимо найти и реализовать лучший способ «фильтровать» рассматриваемые промысловые скважины, чтобы лучше «почувствовать» свойства коллектора и анизотропию проводимости пласта [3]. 
Было бы очень полезно провести математические эксперименты в геолого-гидродинамической модели пласта для планирования такой производственной деятельности и получения ее положительных результатов. Следует отметить, что подобная идея основана на обычных методах анализа одиночных скважин в фильтре нестабильного режима.

\section{Уравнение фильтрации сжимающихся жидкостей в гибком пласте}

\section{Значение}

$\mathrm{k}$ - гидравлическая фильтрация пласта, гидропроницаемость, [cм²], [d]; $\mathrm{h}$-толщина пласта, [см];

$\mu$ - вязкость жидкости в пластовых условиях, [спз];

$p(r, t)$ - давление на расстоянии $\mathrm{r}$ от оси буровой в $\mathrm{t}$ время, [кгГ/см $\left.{ }^{2}\right]$;

$P_{c}$ - забойное давление скважины, [кгГ/см²];

$P_{n л}$ - пластовое давление, [кгГ /см];

$r_{c}$ - эффективный радиус скважины (с учетом скин-эффекта), [см];

$Q(t)$ - дебит скважины в пластовых условиях, $\left[\mathrm{cm}^{3} / \mathrm{c}\right]$;

$\boldsymbol{K}=\frac{\boldsymbol{k} \boldsymbol{h}}{\boldsymbol{\mu}}-$ гидропроницаемость пласта, [дсм/см];

$$
\left.\chi=\frac{\mathrm{k}}{\mu\left(\mathrm{m} \beta_{\text {ж }}+\beta_{\mathrm{c}}\right)}=\frac{\mathrm{k}}{\mu \beta^{*}}-\text { параметр пьезопроницаемости, [дсм }{ }^{2} / \mathrm{cпз}\right] \text {; }
$$

$m$ - пористость пласта ;

$\beta_{\text {ж, }} \quad \beta_{c}$ - коэффициенты упругого объема жидкости и пласта соответственно $\left[\mathrm{cm}^{2} / \kappa \Gamma \Gamma\right]$;

$c$ - приведенный коэффициент упругого объема пласта, [ $\left.\mathrm{cm}^{2} / \kappa \Gamma Г\right]$;

$\operatorname{expint}(x)=-\boldsymbol{E} i(-x)=\int_{x}^{\infty} \int_{u}^{-u} d u-\quad$ функция интегрального индекса.

Предположим, что один бесконечно большой пласт с постоянной толщиной, не пересекающий верхнюю и нижнюю границы, с точки $(\mathrm{x}, \mathrm{y})=(0,0)$ дает продукцию из одной скважины. Если не воздействовать на пласт в начальный момент времени $\mathrm{t}=0$, то распределение давления в последние моменты будет симметрично цилиндрическим $\mathrm{p}(\mathrm{x}, \mathrm{y}, \mathrm{t})=\mathrm{p}(\mathrm{r}, \mathrm{t})$ :

$$
\frac{1}{r} \frac{\partial}{\partial r}\left(r \frac{\partial p}{\partial r}\right)=\frac{1}{\chi} \cdot \frac{\partial p}{\partial t},
$$

и он записывается в виде уравнения теплопроводности $[1,2]$ : 
Где: $\mathrm{r}=\sqrt{x^{2}+y^{2}}, \chi=\frac{k}{\mu\left(m \beta_{6}+\beta_{c}\right)}=\frac{k}{\mu \beta^{*}}$ - коэффициент пъезопроводности; $m$ - пористость пласта;

$\beta_{ж}, \beta_{c}-$ упругие емкостные коэффициенты жидкости и пласта.

$\beta^{*}=m \beta_{ж}+\beta_{c}-$ приведенный упругий емкостный коэффициент пласта.

Кроме формулы (1) еще и давление должно удовлетворить:

$$
p(r, 0)=p_{n л}
$$

первоначальные и краевые условия.

$$
-\frac{2 \pi \mathrm{kh}}{\mu} \cdot\left(r \frac{\partial p}{\partial r}\right)_{r=r}=Q(t), p(r, O) \rightarrow p_{n n}, r \rightarrow \infty
$$

где: $P_{n л}-$ пластовое давление (давление невоздейственного пласта);

$Q(t)$ - дебит скважины в пластовых условиях;

$r_{c}-$ эффективный радиус скважины (с учетом скин эффекта).

М. Маскетом и И.А. Чарнийем [1,2] найдено решение, удовлетворяющее выше приведенные начальные и краевые условия уравнения (1).

$$
p(r, t)=p_{n n}-\frac{\mu}{4 \pi \mathrm{kh}} \cdot \int_{0}^{t} \frac{Q(t-\tau) \cdot \exp \left(-\frac{r_{c}^{2}+r^{2}}{4 \kappa \tau}\right)}{\tau} \cdot I_{o}\left(\frac{r_{c} r}{2 \chi \tau}\right) d \tau
$$

Где: $I_{0}$ - функция Бесселя первого и нулевого порядка с вымышленным аргументом. Учитывая, что точка пуска скважины $\left(r_{c}=0\right)$ равна $I_{0}(0)=1$,

$$
p(r, t)=p_{n, l}-\frac{\mu}{4 \pi \mathrm{kh}} \cdot \int_{0}^{t} \frac{Q(t-\tau)}{\tau} \cdot \exp \left(-\frac{r^{2}}{4 \chi \tau}\right) d \tau
$$

Последнее уравнение является основным уравнением для интерпретации кривой изменения давления (КИД).

Рассмотрим теперь несколько примеров, имеющих практическое значение.

Пример 1. Пусть, $Q(t)=Q_{0}=$ const постоянным выходом (продукции) из скважины. Тогда:

$$
p(r, t)=p_{n \pi}-\frac{\mu \cdot Q_{0}}{4 \pi \mathrm{kh}} \cdot \int_{0}^{t} \frac{1}{\tau} \cdot \exp \left(-\frac{r^{2}}{4 \chi \tau}\right) d \tau=\mathrm{p}_{\text {пл }}-\frac{\mu \cdot Q_{0}}{4 \pi \mathrm{kh}} \cdot \int_{\frac{\mathrm{r}^{2}}{4 x t}}^{\infty} \frac{e^{-u}}{u} \cdot d u .
$$


это уравнение, можно записать следующим образом:

$$
p(r, t)=p_{n л}-\frac{\mu \cdot Q_{0}}{4 \pi \mathrm{kh}} \cdot \operatorname{expint}\left(\frac{r^{2}}{4 \chi \tau}\right) .
$$

Где:

$$
\operatorname{expint}(x)=\int_{x}^{\infty} \frac{e^{-u}}{u} d u, x \geq 0
$$

является функцией с интегральным индексом.

При малых значениях аргумента функция с интегральным индексом (как видно из рисунка №1) имеет следующую асимптотику:

$$
\operatorname{expint}(x) \approx \ln \frac{1}{x}-0,57722, x<<1 .
$$

При этом допущенная погрешность при $x<0.01$ не переходит $0.25 \%$, при $x<0.03$ не переходит 1\%, при $x<0.1$ не переходит 5,7\%, при $x<0,14$ не переходит 9,7\%.

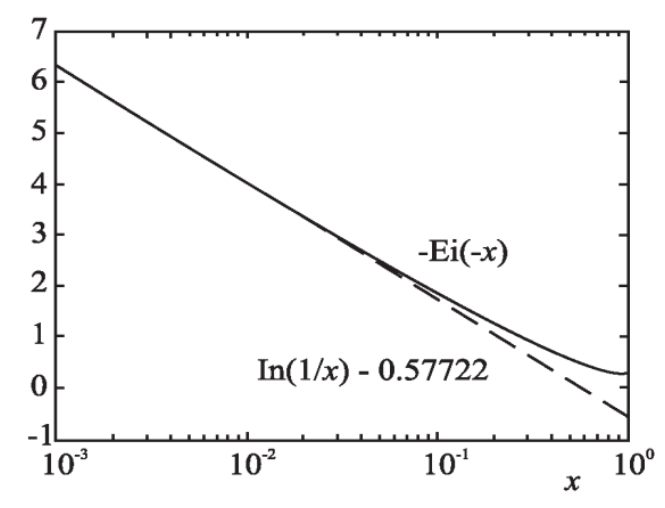

Рисунок №1. Сравнение функции с интегральным индексом ехріnt (x) = Ei (-x) (сплошной кривой) и его аппроксимации ln 1x - 0,57722 (прерывистой кривой) 


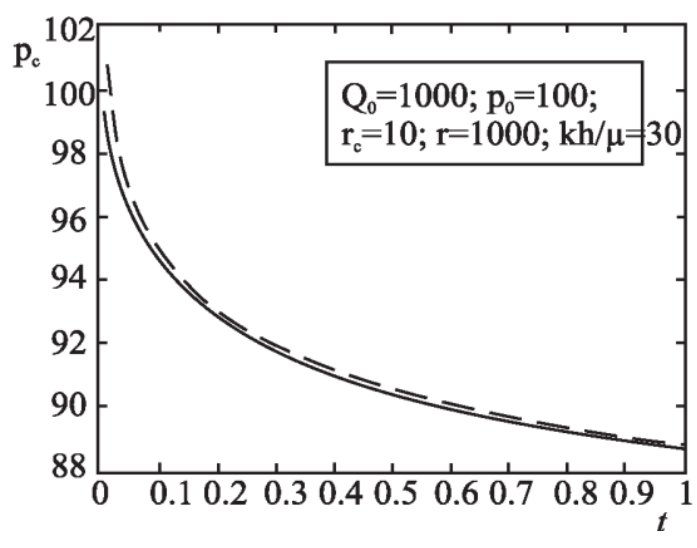

Рисунок № 2. График изменения давления на дне скважины по времени: сплошная кривая - по уравнению (6), прерывистая (штрих) кривая - по уравнению (8)

при, $\frac{r_{c}^{2}}{4 \chi t}<<1$ (для достаточно большего времени) забойное давление скважины можно выражать в виде.

$$
p_{c}(t)=p\left(r_{c}, t\right) \approx p_{n l}-\frac{\mu \cdot Q_{0}}{4 \pi \mathrm{kh}} \cdot\left[\ln \frac{4 \chi \tau}{\mathrm{r}_{c}^{2}}-0.57722\right],
$$

На рисунке №2 представлены кривые зависимости давления в скважине от времени, полученные по уравнениям (6) (сплошная линия) и (прерывистая штрих линия) для следующих нормальных значений: $\chi=1000$ $\mathrm{cm}^{2} / \mathrm{c}, r_{c}=10 \mathrm{cм}, k h / \mu=30$ дсм/спз, $Q=1000 \mathrm{~cm}^{3} / \mathrm{c}, p_{n л}=100$ кгГ $/ \mathrm{cm}^{2}$

Пример 2. Пусть при $0 \leq t \leq T, Q(t)=Q_{0}$, при $t>T, Q(t)=Q_{1}$. Тогда: $t \leq T$ давление определяется уравнением из примера №1, но когда $\mathrm{t}>\mathrm{T}$, мы получаем:

$$
\begin{aligned}
& p(r, t)=p_{n z}-\frac{\mu}{4 \pi \mathrm{kh}} \cdot \int_{0}^{t} \frac{\mathrm{Q}(\mathrm{t}-\tau)}{\tau} \cdot \exp \left(-\frac{r^{2}}{4 \chi \tau}\right) d \tau= \\
& \mathrm{p}_{\text {пи }}-\frac{\mu \cdot Q_{1}}{4 \pi \mathrm{kh}} \cdot \int_{0}^{\mathrm{t}-\mathrm{T}} \frac{1}{\tau} \cdot \exp \left(-\frac{r^{2}}{4 \chi \tau}\right) d \tau-\frac{\mu Q_{0}}{4 \pi \mathrm{kh}} \cdot \int_{\mathrm{t}-\mathrm{\tau}}^{\mathrm{t}} \frac{1}{\tau} \cdot \exp \left(-\frac{r^{2}}{4 \chi \tau}\right) d \tau .
\end{aligned}
$$

Согласно определению функции, с интегральным индексом:

$$
\begin{aligned}
& \int_{t_{1}}^{t_{2}} \frac{1}{\tau} \cdot \exp \left(-\frac{r^{2}}{4 \chi \tau}\right) d \tau=\int_{\frac{r^{2}}{4 t_{2}}}^{\frac{r^{2}}{4 \mathrm{t}_{1}}} \frac{\mathrm{e}^{-\mathrm{u}}}{\mathrm{u}} d u= \\
& \operatorname{expint}\left(\frac{r^{2}}{4 \chi \mathrm{t}_{2}}\right)-\operatorname{expint}\left(\frac{r^{2}}{4 \chi \mathrm{t}_{1}}\right) .
\end{aligned}
$$


Следовательно:

$$
p(r, t)=p_{n z}-\frac{\mu\left(Q_{1}-Q_{0}\right)}{4 \pi \mathrm{kh}} \times\left[\operatorname{expint}\left(\frac{r^{2}}{4 \chi(\mathrm{t}-\mathrm{T})}\right)\right]-\frac{\mu Q_{0}}{4 \pi \mathrm{kh}} \cdot\left[\operatorname{expint}\left(\frac{r^{2}}{4 \chi t)}\right)\right] .
$$

Таким образом, в случае $t>T$ для определения давления в забое скважины оправдано следующее уравнение:

$$
p_{c}(t)=p_{n t}-\frac{\mu\left(Q_{1}-Q_{0}\right)}{4 \pi \mathrm{kh}} \cdot\left[\operatorname{expint}\left(\frac{r_{\mathrm{c}}^{2}}{4 \chi(t-T)}\right)\right]-\frac{\mu Q_{0}}{4 \pi \mathrm{kh}} \cdot\left[\operatorname{expint}\left(\frac{r_{\mathrm{c}}^{2}}{4 \chi t)}\right)\right], t>T
$$

В частности, $Q_{1}=0$ получаем предварительно известное нам уравнение для кривого восстановления забойного давления (КЗД) скважины приостановленной после длительного периода эксплуатации:

$$
p_{c}(t)=p_{n z}-\frac{\mu Q_{o}}{4 \pi \mathrm{kh}} \times\left[\operatorname{expint}\left(\frac{r_{\mathrm{c}}^{2}}{4 \chi t}\right)-\operatorname{expint} \frac{r_{\mathrm{c}}^{2}}{4 \chi(t-T)}\right], t>T .
$$

когда $\frac{r_{c}^{2}}{4 \chi(t-T)} \ll 1$ для функции с интегральным индексом (7) используя аппроксимацию, окончательное уравнение:

$$
p_{c}(t)=p_{n z}-\frac{\mu Q_{0}}{4 \pi \mathrm{kh}} \cdot \ln \left(\frac{t}{t-T}\right), t>T, \frac{r_{\mathrm{c}}^{2}}{4 \chi(t-T)}<<1
$$

можно записать в следующем виде.

Аналогично, для общего случая, даже если $Q_{1}$ в отличии от нуля, в место (10) уравнение $\frac{r_{c}^{2}}{4 \chi(t-T)} \ll 1$ можно использовать следующее более простую аппроксимацию:

$$
p_{c}(t)=p_{n z}-\frac{\mu Q_{o}}{4 \pi \mathrm{kh}} \cdot \ln \left(\frac{t}{t-T}\right)-\frac{\mu Q_{1}}{4 \pi \mathrm{kh}} \cdot \ln \left(\frac{4 \chi(t-T)}{r_{\mathrm{c}}^{2}}\right)+\frac{0.57722 \cdot \mu Q_{1}}{4 \pi \mathrm{kh}}, t>T .
$$

Следующий пример обобщает приведенные выше примеры.

Пример №3. Пусть $0=t_{0}<\mathbf{t}_{1}<\ldots<t_{n}$ - временные последовательности, выход жидкости к моменту $t=0$ будет $Q(t)=0$ и если $t \geq 0$ он будет иметь вид следующей ступенчатой функции: 


$$
Q(t)=\left\{\begin{array}{c}
Q_{0}, t_{0} \leq t<t_{1} \\
Q_{1}, t_{1} \leq t<t_{2} \\
\ldots \\
Q_{n-1}, t_{n-1} \leq t<t_{n} \\
0, \quad t \geq t_{n}
\end{array}\right.
$$

Тогда: Распределение давления за время $\mathrm{t}$, соответствующее временному интервалу $\mathrm{t}_{\mathrm{i}}=\mathrm{t}<\mathrm{t}_{\mathrm{i}+1}$ согласно уравнению (5) примет следующий вид:

$$
\begin{aligned}
& p(r, t)=p_{n z}-\frac{\mu}{4 \pi \mathrm{kh}} \cdot \int_{0}^{t} \frac{\mathrm{Q}(\mathrm{t}-\tau)}{\tau} \cdot \exp \left(-\frac{r^{2}}{4 \chi \tau}\right) d \tau= \\
& =p_{n z}-\frac{\mu \cdot \mathrm{Q}_{\mathrm{i}}}{4 \pi \mathrm{kh}} \cdot \int_{0}^{t-t_{i}} \frac{1}{\tau} \cdot \exp \left(-\frac{r^{2}}{4 \chi \tau}\right) d \tau-\frac{\mu \cdot \mathrm{Q}_{\mathrm{i}-1} \times}{4 \pi \mathrm{kh}} \times \\
& \times \int_{t-t_{i}}^{t-t_{i t}} \frac{1}{\tau} \cdot \exp \left(-\frac{r^{2}}{4 \chi \tau}\right) d \tau-\frac{\mu \cdot \mathrm{Q}_{0}}{4 \pi \mathrm{kh}} \cdot \int_{t-t_{l}}^{t-t_{0}} \frac{1}{\tau} \times \\
& \times \exp \left(-\frac{r^{2}}{4 \chi \tau}\right) d \tau=\mathrm{p}_{\text {пा }}-\frac{\mu \cdot \mathrm{Q}_{\mathrm{i}}}{4 \pi \mathrm{kh}} \cdot\left[-E i\left(-\frac{r^{2}}{4 \chi\left(t-t_{1}\right)}\right)\right]- \\
& -\sum_{\mathrm{j}=0}^{\mathrm{i}-1} \frac{\mu \cdot \mathrm{Q}_{\mathrm{j}}}{4 \pi \mathrm{kh}} \cdot\left[-E i\left(-\frac{r^{2}}{4 \chi\left(t-t_{j}\right)}\right)+E i\left(-\frac{r^{2}}{4 \chi\left(t-t_{j+1}\right)}\right)\right] .
\end{aligned}
$$

Если $\mathrm{t}>\mathrm{tn}$, то получим, аналогичное.

Пример №4. Пусть Т $>0$ и

$$
Q(t)=\left\{\begin{array}{l}
0, \quad t<0 \\
Q_{0}, \quad 0 \leq t<T, \\
Q_{1}+\left(Q_{o}-Q_{1}\right) \cdot e^{\alpha(t-T)}, t \geq T
\end{array}\right.
$$

В этом случае уравнение давления при $\mathrm{t}<\mathrm{T}$ соответствует уравнению в примере 1. Если $\mathrm{t}>\mathrm{T}$, то по уравнению (5) получаем: 


$$
\begin{aligned}
& p(r, t)=p_{n l}-\frac{\mu}{4 \pi \mathrm{kh}} \cdot \int_{0}^{t} \frac{\mathrm{Q}(\mathrm{t}-\tau)}{\tau} \cdot \exp \left(-\frac{r^{2}}{4 \chi \tau}\right) d \tau=p_{n z}-\frac{\mu}{4 \pi \mathrm{kh}} \times \\
& \times \int_{0}^{t} \frac{\mathrm{Q}(\tau)}{(t-\tau)} \cdot \exp \left(-\frac{r^{2}}{4 \chi(t-\tau)}\right) d \tau=p_{n z}-\frac{\mu}{4 \pi \mathrm{kh}} \times \\
& \times \int_{0}^{T} \frac{\mathrm{Q}_{0}}{(t-\tau)} \cdot \exp \left(-\frac{r^{2}}{4 \chi(t-\tau)}\right) d \tau-\frac{\mu}{4 \pi \mathrm{kh}} \cdot \int_{T}^{t} \frac{\mathrm{Q}_{1}}{(t-\tau)} \times \\
& \times \exp \left(-\frac{r^{2}}{4 \chi(t-\tau)}\right) d \tau-\frac{\mu \cdot\left(\mathrm{Q}_{0}-\mathrm{Q}_{1}\right)}{4 \pi \mathrm{kh}} \cdot \int_{T}^{t} \frac{\mathrm{e}^{\mathrm{aj}(\tau-\mathrm{T})}}{(t-\tau)} \times \\
& \times \exp \left(-\frac{r^{2}}{4 \chi(t-\tau)}\right) d \tau=p_{n s}-\frac{\mu\left(\mathrm{Q}_{1}-\mathrm{Q}_{0}\right)}{4 \pi \mathrm{kh}} \times \\
& \times\left[-E i\left(-\frac{r^{2}}{4 \chi(t-T)}\right)\right]-\frac{\mu \mathrm{Q}_{0}}{4 \pi \mathrm{kh}} \cdot\left[-E i\left(-\frac{r^{2}}{4 \chi t}\right)\right]- \\
& -\frac{\mu \cdot\left(\mathrm{Q}_{0}-\mathrm{Q}_{1}\right)}{4 \pi \mathrm{kh}} \cdot \int_{T}^{t} \frac{\mathrm{e}^{-\alpha(\tau-\mathrm{T})}}{(t-\tau)} \cdot \exp \left(-\frac{r^{2}}{4 \chi(t-\tau)}\right) d \tau .
\end{aligned}
$$

Таким образом, при $t>T$ и $\frac{r_{c}^{2}}{4 \chi(t-T)} \ll<1$ исходя из уравнения, получаем:

$$
\begin{aligned}
& p_{c}(t)=p_{n \tau}-\frac{\mu \mathrm{Q}_{0}}{4 \pi \mathrm{kh}} \cdot \log \left(\frac{\mathrm{t}}{\mathrm{t}-\mathrm{T}}\right)-\frac{\mu \mathrm{Q}_{1}}{4 \pi \mathrm{kh}} \cdot \log \left(\frac{4 \chi(\mathrm{t}-\mathrm{T})}{r_{c}^{2}}\right)+ \\
& +\frac{0.57722 \cdot \mu \mathrm{Q}_{1}}{4 \pi \mathrm{kh}}-\frac{\mu \cdot\left(\mathrm{Q}_{0}-\mathrm{Q}_{1}\right)}{4 \pi \mathrm{kh}} \times \int_{T}^{t} \frac{\mathrm{e}^{-\alpha(\tau-\mathrm{T})}}{(t-\tau)} \cdot \exp \left(-\frac{r_{c}^{2}}{4 \chi(t-\tau)}\right) d \tau .
\end{aligned}
$$

Пример №5. Пусть $\delta T>0$ и

$$
Q(t)=\left\{\begin{array}{l}
0, \quad t<0 . \\
\frac{Q_{0}}{\delta T} \cdot t, \quad 0 \leq t<\delta T, \\
Q_{0}, t \geq \delta T .
\end{array}\right.
$$


Тогда: при $t>\delta T$ будет:

$$
\begin{aligned}
& p(r, t)=p_{n z}-\frac{\mu}{4 \pi \mathrm{kh}} \cdot \int_{0}^{\mathrm{t}} \frac{\mathrm{Q}(\tau)}{(t-\tau)} \cdot \exp \left(-\frac{r^{2}}{4 \chi(t-\tau)}\right) d \tau= \\
& p_{n z}-\frac{\mu}{4 \pi \mathrm{kh}} \cdot \frac{\mathrm{Q}_{0}}{\delta \mathrm{T}} \cdot \int_{0}^{\delta \mathrm{T}} \frac{\tau}{(t-\tau)} \cdot \exp \left(-\frac{r^{2}}{4 \chi(t-\tau)}\right) d \tau- \\
& -\frac{\mu \mathrm{Q}_{0}}{4 \pi \mathrm{kh}} \cdot \int_{\delta \mathrm{T}}^{\mathrm{t}} \frac{1}{(t-\tau)} \cdot \exp \left(-\frac{r^{2}}{4 \chi(t-\tau)}\right) d \tau .
\end{aligned}
$$

Теперь давайте посчитаем каждое расстояние отдельно. Для более общего случая, когда $0 \leq t_{1} \leq t_{2} \leq \mathrm{t}$ получаем:

$$
\begin{aligned}
& \int_{t_{1}}^{t_{2}} \frac{1}{(t-\tau)} \cdot \exp \left(-\frac{r^{2}}{4 \chi(t-\tau)}\right) d \tau=-E i\left(-u_{1}\right)+E i\left(-u_{2}\right), \\
& u_{1}=\frac{r^{2}}{4 \chi\left(t-t_{i}\right)}, i=1,2 \\
& \int_{t_{1}}^{t_{2}} \exp \left(-\frac{r^{2}}{4 \chi(t-\tau)}\right) d \tau=\int_{u_{1}}^{u_{2}} e^{-u} \cdot \frac{r^{2}}{4 \chi u^{2}} d u=\frac{r^{2}}{4 \chi} \cdot \int_{u_{1}}^{u_{2}} \frac{e^{-u}}{u^{2}} d u= \\
& =-\frac{r^{2}}{4 \chi} \cdot \int_{u_{1}}^{u_{2}} e^{-u} d\left(\frac{1}{u}\right)=\frac{r^{2}}{4 \chi} \cdot\left[-\left.\frac{e^{-u}}{u}\right|_{u_{1}} ^{u_{2}}-\int_{u_{1}}^{u_{2}} \frac{e^{-u}}{u} d u\right]= \\
& =\frac{r^{2}}{4 \chi} \cdot\left[-\frac{e^{-u_{I}}}{u_{1}}-\frac{e^{-u_{2}}}{u_{2}}+E i\left(-\mathrm{u}_{1}\right)-E i\left(-\mathrm{u}_{2}\right)\right] .
\end{aligned}
$$


Следовательно:

$$
\begin{aligned}
& \int_{0}^{\delta \mathrm{T}} \frac{\tau}{(t-\tau)} \cdot \exp \left(-\frac{r^{2}}{4 \chi(t-\tau)}\right) d \tau=\int_{0}^{\delta \mathrm{T}} \frac{(\tau-t)+t}{(t-\tau)} \times \\
& \times \exp \left(-\frac{r^{2}}{4 \chi(t-\tau)}\right) d \tau=-\int_{0}^{\delta \mathrm{T}} \exp \left(-\frac{r^{2}}{4 \chi(t-\tau)}\right) d \tau+ \\
& +t \cdot \int_{0}^{\delta \mathrm{T}} \frac{1}{(t-\tau)} \cdot \exp \left(-\frac{r^{2}}{4 \chi(t-\tau)}\right) d \tau=\frac{r^{2}}{4 \chi} \times \\
& \times\left\{\frac{\exp \left(-\frac{r^{2}}{4 \chi(t-\delta T)}\right)}{\frac{r^{2}}{4 \chi(t-\delta T)}}-\frac{\exp \left(-\frac{r^{2}}{4 \chi t}\right)}{\frac{r^{2}}{4 \chi t}}+E i\left(-\frac{r^{2}}{4 \chi(t-\delta T)}\right)-\right. \\
& \left.-E i\left(-\frac{r^{2}}{4 \chi t}\right)\right\}+t \cdot\left\{-E i\left(-\frac{r^{2}}{4 \chi t}\right)+E i\left(-\frac{r^{2}}{4 \chi(t-\delta T)}\right)\right\} \\
& \int_{\delta \mathrm{T}}^{t} \frac{1}{(t-\tau)} \cdot \exp \left(-\frac{r^{2}}{4 \chi(t-\tau)}\right) d \tau=-E i\left(-\frac{r^{2}}{4 \chi(t-\delta T)}\right)
\end{aligned}
$$

Таким образом, при $\mathrm{t}>T$ получаем следующее уравнение для выхода (18):

$$
\begin{aligned}
& p(r, t)=p_{n z}-\frac{\mu}{4 \pi \mathrm{kh}} \cdot \frac{\mathrm{Q}_{0}}{\delta \mathrm{T}} \cdot \int_{0}^{\delta \mathrm{T}} \frac{\tau}{(t-\tau)} \cdot \exp \left(-\frac{r^{2}}{4 \chi(t-\tau)}\right) d \tau-\frac{\mu \mathrm{Q}_{0}}{4 \pi \mathrm{kh}} \\
& \cdot \int_{\delta \mathrm{T}}^{1} \frac{1}{(t-\tau)} \cdot \exp \left(-\frac{r^{2}}{4 \chi(t-\tau)}\right) d \tau=p_{n z}-\frac{\mu \mathrm{Q}_{0}}{4 \pi \mathrm{kh}} \cdot\left[-E i\left(-\frac{r^{2}}{4 \chi(t-\delta T)}\right)\right] \\
& -\frac{\mu \mathrm{Q}_{0}}{4 \pi \mathrm{kh}} \cdot \frac{t}{\delta T} \times\left\{-E i\left(-\frac{r^{2}}{4 \chi t)}\right)+E i\left(-\frac{r^{2}}{4 \chi(t-\delta T)}\right)-\frac{\mu \mathrm{Q}_{0}}{4 \pi \mathrm{kh}} \cdot \frac{r^{2}}{4 \chi} \cdot \frac{1}{\delta T} \times\right. \\
& \times\left\{\frac{\exp \left(-\frac{r^{2}}{4 \chi(t-\delta T)}\right)}{\frac{r^{2}}{4 \chi(t-\delta T)}}-\frac{\exp \left(-\frac{r^{2}}{4 \chi t}\right)}{E i\left(-\frac{r^{2}}{4 \chi(t-\delta T)}\right)-\frac{r^{2}}{4 \chi\left(-\frac{r^{2}}{4 \chi t}\right)}}\right\}
\end{aligned}
$$


Если $t \leq \delta T$ тогда аналогично прежнему уравнению получаем:

$$
\begin{aligned}
& p(r, t)=p_{n z}-\frac{\mu}{4 \pi \mathrm{kh}} \cdot \frac{\mathrm{Q}_{0}}{\delta \mathrm{T}} \cdot \int_{0}^{t} \frac{\tau}{(t-\tau)} \cdot \exp \left(-\frac{r^{2}}{4 \chi(t-\tau)}\right) d \tau= \\
& =p_{n z}-\frac{\mu \mathrm{Q}_{0}}{4 \pi \mathrm{kh}} \cdot \frac{\mathrm{t}}{\delta \mathrm{T}} \cdot\left\{-E i\left(-\frac{r^{2}}{4 \chi t}\right)\right\}-\frac{\mu \mathrm{Q}_{0}}{4 \pi \mathrm{kh}} \cdot \frac{r^{2}}{4 \chi \delta T} \times \\
& \times\left\{\frac{\exp \left(-\frac{r^{2}}{4 \chi t}\right)}{\frac{r^{2}}{4 \chi t}}-E i\left(-\frac{r^{2}}{4 \chi t}\right)\right\}
\end{aligned}
$$

(21b)

Пример №6. Пусть $\mathbf{t}_{1}<\mathbf{t}_{2}$, и

$$
Q(t)=\left\{\begin{array}{c}
0, t<t_{1}, \\
\frac{\left(Q_{2}-Q_{1}\right)}{\left(t_{2}-t_{1}\right)} \cdot\left(t-t_{1}\right), t_{1} \leq t<t_{2}, \\
Q_{0}, t \geq t_{2},
\end{array}\right.
$$

тогда: при $t \leq t_{1} p(r, t)=p_{n л}$. Если $t_{1} \leq t \leq t_{2}$, то

$$
a=Q_{1}-\frac{\left(Q_{2}-Q_{1}\right)}{\left(t_{2}-t_{1}\right)} \cdot t_{1}, \quad b=\frac{\left(Q_{2}-Q_{1}\right)}{\left(t_{2}-t_{1}\right)}
$$

вводя значения, получаем:

$$
\begin{aligned}
& p(r, t)=p_{n z}-\frac{\mu}{4 \pi \mathrm{kh}} \cdot \int_{\mathrm{t}_{1}}^{\mathrm{t}} \frac{\mathrm{Q}(\tau)}{(t-\tau)} \cdot \exp \left(-\frac{r^{2}}{4 \chi(t-\tau)}\right) d \tau= \\
& p_{n z} \frac{\mu}{4 \pi \mathrm{kh}} \cdot \int_{\mathrm{t}_{1}}^{\mathrm{t}} \frac{\mathrm{a}+\mathrm{b} \tau}{(t-\tau)} \cdot \exp \left(-\frac{r^{2}}{4 \chi(t-\tau)}\right) d \tau= \\
& p_{n z} \frac{\mu \cdot(\mathrm{a}+\mathrm{b} t)}{4 \pi \mathrm{kh}} \cdot \int_{\mathrm{t}_{1}}^{\mathrm{t}} \frac{1}{(t-\tau)} \cdot \exp \left(-\frac{r^{2}}{4 \chi(t-\tau)}\right) d \tau+ \\
& +\frac{\mu \cdot \mathrm{b}}{4 \pi \mathrm{kh}} \cdot \int_{\mathrm{t}_{1}}^{\mathrm{t}} \exp \left(-\frac{r^{2}}{4 \chi(t-\tau)}\right) d \tau
\end{aligned}
$$


Теперь, используя уравнения (19) и (20), получаем для времени $t_{1} \leq t \leq t_{2}$ :

$$
\begin{aligned}
& p(r, t)=p_{n z}-\frac{\mu(\mathrm{a}+\mathrm{b} t)}{4 \pi \mathrm{kh}} \cdot \int_{\mathrm{t}_{1}}^{\mathrm{t}} \frac{1}{(t-\tau)} \cdot \exp \left(-\frac{r^{2}}{4 \chi(t-\tau)}\right) d \tau+ \\
& +\frac{\mu \cdot \mathrm{b}}{4 \pi \mathrm{kh}} \cdot \int_{t_{1}}^{\mathrm{t}} \exp \left(-\frac{r^{2}}{4 \chi(t-\tau)}\right) d \tau=p_{n z} \frac{\mu(\mathrm{a}+\mathrm{b} t)}{4 \pi \mathrm{kh}} \times \\
& \times \operatorname{expint}\left(-\frac{r^{2}}{4 \chi t\left(t-t_{1}\right)}\right)+\frac{\mu \cdot \mathrm{b}}{4 \pi \mathrm{kh}} \cdot-\frac{r^{2}}{4 \chi} \times \\
& \times\left[\frac{\exp \left(-\frac{r^{2}}{4 \chi\left(t-t_{1}\right)}\right)}{\frac{r^{2}}{4 \chi t\left(t-t_{1}\right)}}-\operatorname{expint}\left(-\frac{r^{2}}{4 \chi t\left(t-t_{1}\right)}\right)\right]
\end{aligned}
$$

Если $\mathrm{t}_{1}>\mathrm{t}_{2}$ то мы получаем указанную выше аналогию:

Пример №7. Пусть $\mathbf{T}>\delta T_{1}>0$, и $\delta T_{2}>0$,

$$
Q(t)=\left\{\begin{array}{l}
0, t<0, \\
\frac{Q_{1}}{\delta T_{1}} \cdot t, \quad 0 \leq t<\delta T_{1}, Q_{1}, \delta T_{1} \leq t<T, \\
Q_{1}+\frac{\left(Q_{2}-Q_{1}\right)}{\delta T_{2}} \cdot(t-T), \quad T \leq t<T+\delta T_{2} \\
Q_{2}, t \geq T+\delta T_{2} .
\end{array}\right.
$$

Полученное на основании расчетов схематическое изображение изменения выхода показано на рисунке №3.

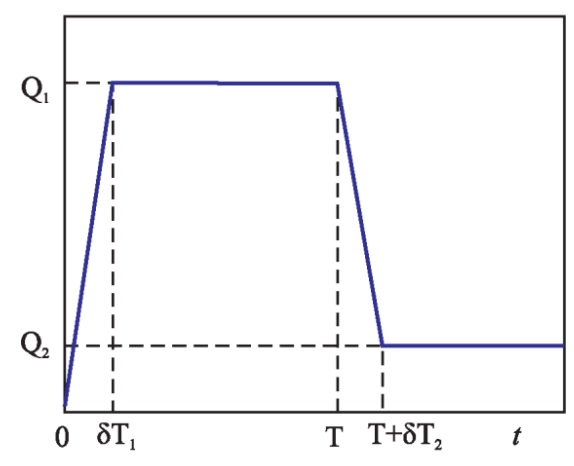

Рисунок №3. График выхода

Определение (идентификация) параметров газовых коллекторов 
В фильтрации идеального газа уравнение для давления отличается от уравнения жидкостей (1), а данным отличием будет квадрат давления вместо давления, а вместо пьезопроводимости $\bar{\chi}=\frac{k p_{?}}{\mu m_{0}} \quad$ будет параметр [2].

$\frac{1}{\mathrm{r}} \frac{\partial}{\partial \mathrm{r}}\left(\mathrm{r} \frac{\partial \mathrm{p}^{2}}{\partial \mathrm{r}}\right)-\frac{1}{\chi} \cdot \frac{\partial \mathrm{p}^{2}}{\partial \mathrm{t}}$

Где: $m_{0}-$ коэффициент пористости;

$k-$ проницаемость пласта;

$\mu \quad-$ вязкость газа;

$p_{n л}-$ давление в невоздейственном пласте.

В этом случае начальное условие записывается следующим образом:

$$
\mathrm{p}^{2}(r, 0)=p_{n л} \text {. }
$$

Для идеального газа вместо побочного условия (3) используется следующее соотношение [2].

$$
-\frac{2 \pi \mathrm{kh}}{\mu} \cdot\left(\mathrm{r} \frac{\partial \mathrm{p}^{2}}{\partial \mathrm{r}}\right)_{\mathrm{r}=\mathrm{r}_{\mathrm{c}}}=2 \cdot \mathrm{p}_{\mathrm{aT}} \cdot \mathrm{Q}_{\mathrm{aT}}(\mathrm{t}) .
$$

Где: $p_{a m}-$ атмосферное давление;

$Q_{a m}$ - выход в устье скважины. Граничное условие бесконечности примет следующий вид:

$$
p^{2}(r . t)=p_{n l}^{2}, r \rightarrow \propto
$$

Таким образом, в случае определения параметров идеального газового коллектора на основе гидродинамического анализа скважин можно использовать описанную теорию упругих жидкостей, но вместо давления необходимо брать его квадрат и вместо выхода из забоя скважины $2 \cdot p_{a m} \cdot Q_{a m}$ $(t)$.

\section{Список литературы}

1. Азиз Х., Сеттари Э. Математическое моделирование пластовых систем. - М.: Недра, 1982, стр.

2. Басниев К.С., Власов А.М., Кочина И.Н. Максимов В.М. Подземная гидравлика. - М.: Недра, 1986, стр. 303.

3. Гурбанмырадов О.А., Эседуллаев Р.Э., Дурдыев Н.Т., А.Р. Деряев. Гидродинамические проблемы, возникающие при бурении нефтегазовых скважин и способы их решения. - А.: Ылым, 2018, стр.336. 